\title{
Wash your eyes with soap - compulsive ritual or self-injurious behavior?
}

A.V. Costa ${ }^{1}$, N. Trovão², J. Perestrelo², S. Melo ${ }^{1}$, S. Borges ${ }^{1}$, S. Pereira ${ }^{2}$, A. Horta ${ }^{2}$, L. Pires², C. Grifo², D. Leite ${ }^{1,2}$, R. Ribeiro-Silva ${ }^{2}$ ${ }^{1}$ Hospital Center of Vila Nova de Gaia/Espinho, Child and Adolescent Psychiatry, Vila Nova de Gaia, Portugal. ${ }^{2}$ Hospital Center of Vila Nova de Gaia/Espinho, Psychiatry and Mental Health, Vila Nova de Gaia, Portugal.

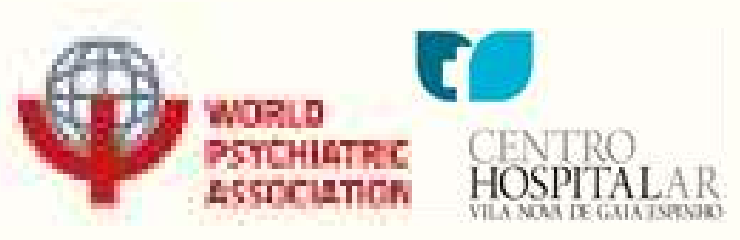

\section{Background:}

The symptomatic presentation of borderline personalities is quite heterogenic given the inability of personality cohesion and immature identity construct. Often obsessive compulsive behaviors arise, being part of the patient identity and providing a sense of continuity, avoiding the disintegration of the self.

This case presentation focuses on deliberate self-harm: washing eyes with soap presenting in a form of obsessive compulsive behavior in a young girl with borderline personality disorder.

Studies reveal an awareness of the detrimental effect of having siblings with serious illnesses and deficiencies (specially with significant behavior problems) conditioned by difficulties in interfamily/sibling relationships and when caregivers may not be available to meet the healthy children basic emotional needs.

\section{Clinical case summary:}

\section{Objectives and Methods:}

Clinical case description for reflection about the symptomatic presentation framed in the context and personality development.

Bibliographic exploration regarding the impact of chronic disease on siblings and clinical presentation of borderline states with repetitive behaviors (namely self-injurious behavior) that organize the affective instability.

A 20-year girl referred for the therapeutic group of our partial hospitalization care by hand-washing rituals (with phisical injury). The provided clinical information is sometimes incongruent, given its immature personality constitution and ill-defined identity construct.

Inserted in a mentally dependent family: older sibling with diagnosis of autism disorder with severe behavioral changes (now stabilized, referring to herself as being currently uncontrolled) and mother with excessive search for her in psychiatric childhood care (since 6 years) refusing all suggested therapeutic proposals. Her parents have been divorced since she was 11 years old. She maintains very sporadic and telephone contacts with her father after the divorce.

She presents herself dissatisfied with occupational situation, dependent on the mother for basic care, directing anger to the teachers and with external locus of control.

The entry into therapeutic group was disrupted with worsening behavior of cleaning, first hands, then the lips (at the same time that she receded and fell asleep) and later the eyes with soap. Such behavior arose in the identification with the group, since the group was constituted by elements with deformities in the eyes, even the nurse have a hordeolum (self-harming in order to insert in herself physical characteristics of the remaining elements); just as at home repetitive behaviors first appeared in childhood as did the brother of the autism spectrum (only way to be seen and loved).

As she moves away from her nurse's gaze, and enters the group, she feels increasingly abandoned, lacking in entertainment and activities testing the prohibitions on escalating aggressive behavior toward the new mother (nurse) and hospital. When was imposed more rigid limits, the necessity of the cleaning rituals ceased.

She did not stay in the group, since after the initial aggravation with anger directed to the hospital, she improved behaviorally not withstanding the conflict of loyalty towards the mother who rivaled the nurse who was taking the place of the good maternal care and affection.

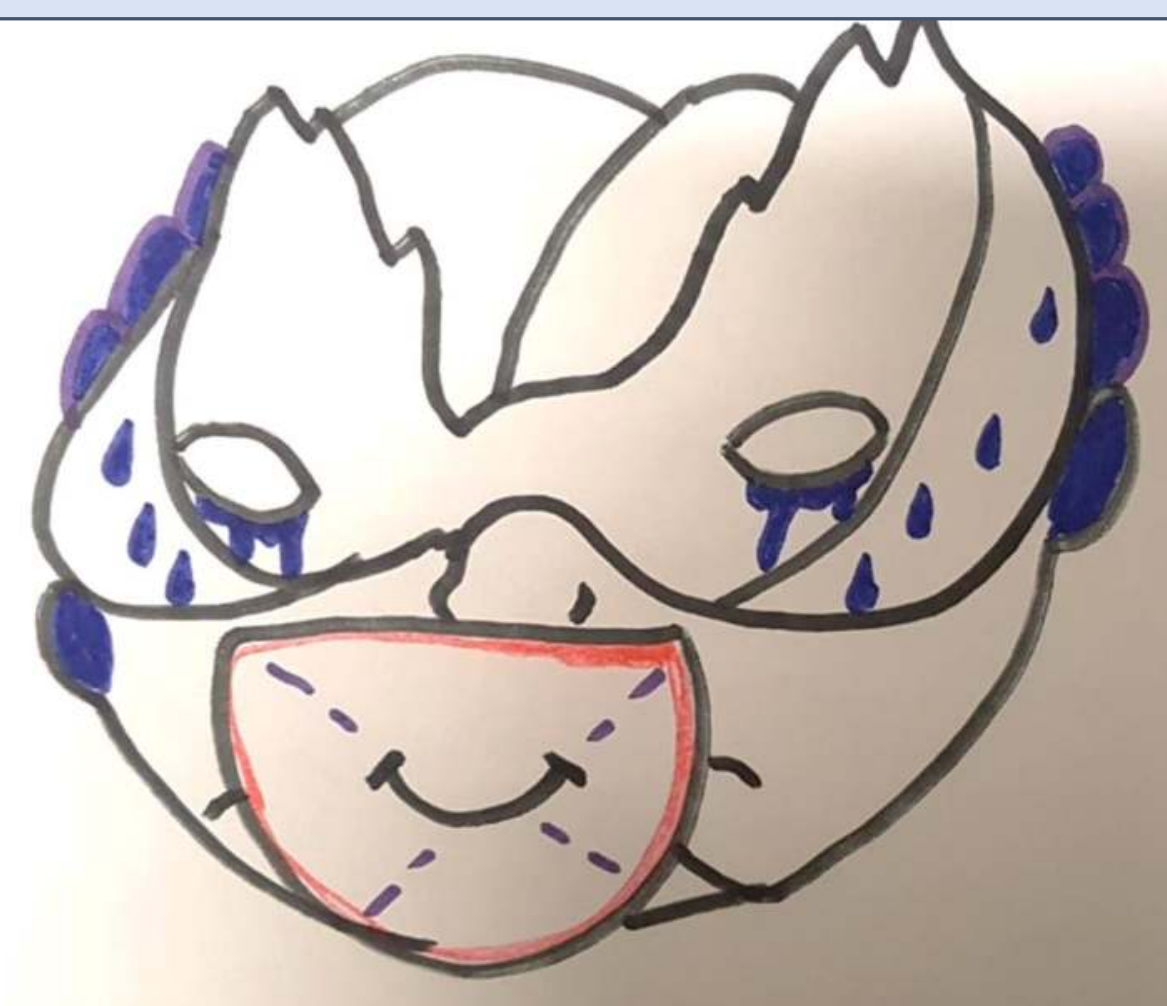

"My mask: mask wrapped in layers, crying abundantly, but that will have to show a smile".

\section{Discussion and}

\section{Conclusions:} disintegration.
"My Coat of Arms: A ring wrapped in a rough rock diamond that needs to be shaped", expressing the need for containment, with ambivalence.

In this clinical case it is denoted the insufficiency internal/external separation, self/object and archaic impulses, disorder that can be organized in obsessive symptoms that require the collaboration of a maternal element for care and to whom aggressiveness is directed. Emotional experiences are corrected in obsessive compulsive behaviors, organizers of internal psychological states - creating a sense of security and control in which actions and thoughts (belonging to one's own) prevent cleavage and

We denote also chronic disease in fratria as a stressor for dysharmonic psycho-affective developments when there is no capacity for emotional support from the system. 